\title{
Comparação entre Ventilação Controlada a Volume e a Pressão no Tratamento da Hipoxemia no Período Pós-Operatório de Cirurgia de Revascularização do Miocárdio *
}

\section{Comparison between Pressure Controlled and Controlled Mandatory Ventilation in the Treatment of Postoperative Hypoxemia after Myocardial Revascularization}

\author{
Fábio Bonini Castellana ${ }^{1}$; Luiz Marcelo Sá Malbouisson, TSA ${ }^{2}$; Maria José Carvalho Carmona, TSA ${ }^{3}$;
} Célia Regina Lopes ${ }^{4}$; José Otávio Costa Auler Júnior, TSA ${ }^{5}$

\begin{abstract}
RESUMO
Castellana FB, Malbouisson LMS, Carmona MJC, Lopes CR, Auler Jr JOC - Comparação entre Ventilação Controlada a Volume e a Pressão no Tratamento da Hipoxemia no Período Pós-Operatório de Cirurgia de Revascularização do Miocárdio
\end{abstract}

JUSTIFICATIVA E OBJETIVOS: Ventilação controlada à pressão tem sido utilizada como modalidade ventilatória de eleição em pacientes que desenvolvem hipoxemia importante no período pós-operatório de cirurgia de revascularização do miocárdio. Contudo não existem estudos mostrando que a ventilação controlada à pressão é mais efetiva na reversão da hipoxemia pós-operatória que ventilação controlada a volume. O objetivo deste estudo foi comparar os efeitos de ventilação controlada à pressão e ventilação controlada a volume sobre a oxigenação sistêmica em pacientes que desenvolvem hipoxemia caracterizada por uma relação $\mathrm{PaO}_{2} / \mathrm{FiO}_{2}$ menor que 200 no período pós-operatório imediato de cirurgia cardíaca.

MÉTODO: Sessenta e um pacientes com relação $\mathrm{PaO}_{2} / \mathrm{FiO}_{2}$ menor que 200 foram alocados em um grupo submetido à ventilação controlada a pressão e outro a volume. O volume corrente, a freqüência respiratória, a relação inspiração/ expiração e a pressão positiva ao final da expiração foram as mesmas no dois grupos. Após a admissão na UTI e após períodos de 1 ou 2 horas de ventilação mecânica, a relação $\mathrm{PaO}_{2} / \mathrm{FiO}_{2}$ e o shunt pulmonar foram quantificados.

\footnotetext{
* Recebido da (Received from) Unidade de Terapia Intensiva Cirúrgica do Instituto do Coração do Hospital das Clínicas da Faculdade de Medicina da Universidade de São Paulo (HC-FMUSP), São Paulo, SP

1. Graduado pela Faculdade de Medicina da USP. Bolsista de Iniciação Científica. Processo FAPESP número: 99/11405-2

2. Assistente da Anestesia e UTI Cirúrgica, Serviço de Anestesiologia, Instituto do Coração, HC-FMUSP

3. Supervisor da Anestesia e UTI Cirúrgica, Serviço de Anestesiologia, Instituto do Coração, HC-FMUSP

4. Fisioterapeuta, Serviço de Fisioterapia, Instituto do Coração, HC-FMUSP

5. Professor Titular da Disciplina de Anestesiologia da FMUSP, Diretor do Serviço de Anestesiologia e UTI Cirúrgica, Instituto do Coração, HC-FMUSP
}

Endereço para Correspondência (Correspondence to)

Dr. Luiz Marcelo Sá Malbouisson

Av. Enéas de Carvalho Aguiar $n^{\circ} 44$ Cerqueira César

05403-000 São Paulo, SP

E-mail: auler@hcnet.usp.br

Apresentado em (Submitted) 30 de agosto de 2002

Aceito (Accepted) para publicação em 17 de dezembro de 2002

(C) Sociedade Brasileira de Anestesiologia, 2003
RESULTADOS: Houve um aumento significativo na relação $\mathrm{PaO}_{2} / \mathrm{FiO}_{2}$ e uma diminuição significativa no shunt pulmonar após 1 ou 2 horas de ventilação mecânica; contudo não foram observadas diferenças entre as modalidades ventilatórias.

CONCLUSÕES: As modalidades ventilatórias controladas a volume e pressão foram igualmente eficientes no tratamento da hipoxemia observada em pacientes no pós-operatório imediato de cirurgia de revascularização do miocárdio, mostrando que o padrão de administração do fluxo inspiratório é pouco relevante para o tratamento da hipoxemia pós-operatória.

Unitermos: CIRURGIA, Cardíaca: revascularização do miocárdio; COMPLICAÇÕES: hipóxia; VENTILAÇÃO: controlada.

\section{SUMMARY}

Castellana FB, Malbouisson LMS, Carmona MJC, Lopes CR, Auler Jr JOC - Comparison between Pressure Controlled and Controlled Mandatory Ventilation in the Treatment of Postoperative Hypoxemia after Myocardial Revascularization

BACKGROUND AND OBJECTIVES: Pressure controlled ventilation (PCV) has been used as the ventilation mode of choice in coronary artery bypass graft surgery patients who develop severe hypoxemia in the immediate postoperative period. However, there are no evidences showing that pressure controlled ventilation is more effective in reversing postoperative hypoxemia than controlled mandatory ventilation (CMV). This study aimed at comparing the effects of both ventilation modes on systemic oxygenation in cardiac surgery patients who develop hypoxemia characterized by $\mathrm{PaO}_{2} / \mathrm{FiO}_{2}$ ratio lower than 200 in the immediate postoperative period.

METHODS: Participated in this study 61 consecutive patients with $\mathrm{PaO}_{2} / \mathrm{FiO}_{2}$ ratio lower than 200 who were randomly allocated in two groups according to the ventilatory mode (CMV and $P C V$ ). Tidal volume, respiratory rate, inspiration/expiration ratio and positive end-expiratory pressure were kept constant throughout the study in both groups. $\mathrm{PaO}_{2} / \mathrm{FiO}_{2}$ ratio and pulmonary shunt were obtained 1 or 2 hours after ICU admission.

RESULTS: A significant increase in $\mathrm{PaO}_{2} / \mathrm{FiO}_{2}$ ratio and a decrease in pulmonary shunt were observed in both groups one or two hours after mechanical ventilation. However, no differences were observed between both ventilatory modes.

CONCLUSIONS: Both ventilatory modes were equally effective in reversing hypoxemia observed in the immediate cardiac surgery postoperative period. Results show that inspiratory flow patterns are not relevant in the treatment of post cardiac surgery hypoxemia.

Key Words: COMPLICATIONS: hypoxemia; SURGERY, Cardiac: coronary artery bypass; VENTILATION: controlled 


\section{INTRODUÇÃO}

№ período pós-operatório imediato de cirurgia para reascularização do miocárdio com circulação extracorpórea, a hipoxemia importante caracterizada por relação $\mathrm{PaO}_{2} / \mathrm{FiO}_{2}$ menor que 200 representa uma complicação de morbidade elevada, que necessita manutenção do suporte ventilatório com pressão positiva ao final da expiração (PEEP) em níveis progressivos ${ }^{1}$, por períodos mais prolongados. O aumento do tempo de ventilação mecânica está relacionado com um aumento na incidência de pneumonias associadas à ventilação mecânica ${ }^{2,3}$. Em nossa Unidade de Terapia Intensiva (UTI), ventilação controlada a pressão (VCP) tem sido utilizada como modalidade ventilatória de eleição frente a quadros de importante hipoxemia no período pós-operatório. Isto fundamenta-se em que esta modalidade ventilatória associa-se a uma recuperação precoce das propriedades mecânicas do sistema respiratório em comparação com a ventilação controlada a volume (VCV), durante a síndrome da angústia respiratória aguda (SARA) ${ }^{4}$ e, conseqüentemente, com a melhora da oxigenação. Outros estudos, porém, não mostraram melhora na oxigenação ou redução no shunt pulmonar verdadeiro na VCP em comparação com a VCV ${ }^{5,6}$.

Durante a fase inspiratória da VCP, o fluxo de gases é livre e diminui de maneira logarítmica quando a pressão das vias aéreas atinge um valor predeterminado durante a insuflação pulmonar. Esta modalidade apresentaria vantagens teóricas como a imitação da pressão de plateau nas vias aéreas levando à menor incidência de barotrauma e, por conseqüência, de lesão pulmonar induzida pela ventilação mecânica no parênquima pulmonar ${ }^{7-11}$ e distribuição mais homogênea do volume corrente, respeitando as complacências regionais.

O objetivo deste estudo é comparar a evolução da oxigenação durante o período pós-operatório imediato em pacientes submetidos à revascularização do miocárdio ventilados nas modalidades VCP ou VCV, com fluxo desacelerado.

\section{MÉTODO}

Após aprovação do Conselho de Ética em Pesquisa do Hospital das Clínicas da Universidade de São Paulo, 61 pacientes submetidos à cirurgia de revascularização do miocárdio com circulação extracorpórea foram estudados. Os critérios de inclusão no estudo foram: 1) relação $\mathrm{PaO}_{2} / \mathrm{FiO}_{2}$ menor que 200 , observada na primeira gasometria arterial obtida no momento da admissão do paciente à unidade de terapia intensiva, 2) ausência de doença pulmonar prévia, 3) ausência de importante disfunção miocárdica, e 4) ausência de fístula broncopleural. Os critérios de exclusão foram: 1) choque circulatório pós-operatório com necessidade de noradrenalina ou dobutamina em doses maiores que $10 \mu \mathrm{g} \cdot \mathrm{kg}^{-1} \cdot \mathrm{min}^{-1}, 2$ ) necessidade de suporte circulatório mecânico, e 3) sangramento pós-operatório importante. O consentimento para participação do paciente no estudo foi obtido após a admissão do mesmo à UTI junto ao responsável.

Todos os pacientes foram monitorizados para o procedimento cirúrgico com eletrocardioscopia, cateter arterial radial e pulmonar, oximetria de pulso, temperatura esofageana e capnografia. Midazolam, fentanil, bloqueador neuromuscular não despolarizante e isoflurano em dose adequadas foram utilizados para indução e manutenção da anestesia. A circulação extracorpórea foi realizada em normotermia e com oxigenador de membrana. Durante a circulação extracorpórea foi utilizada infusão de propofol para manutenção da hipnose e amnésia. Dobutamina, dopamina, nitroglicerina ou nitroprussiato de sódio foram utilizados para saída de circulação extracorpórea conforme a necessidade.

Após a admissão à UTI, uma gasometria arterial foi colhida em cada paciente. Aqueles com relação $\mathrm{PaO}_{2} / \mathrm{FiO}_{2}$ menor que 200 foram alocados aleatoriamente em dois grupos. Um grupo foi ventilado com volume corrente controlado (VCV) com fluxo inspiratório constante e o outro grupo foi ventilado à pressão controlada (PCV), utilizando ventiladores mecânicos. Em ambos os grupos, o volume corrente foi ajustado para $7 \mathrm{ml} . \mathrm{kg}^{-1}$, a freqüência respiratória foi mantida em 12 ipm e a pressão positiva ao final da expiração foi mantida em $5 \mathrm{cmH}_{2} \mathrm{O}$. O tempo inspiratório representava $33 \%$ do tempo total do ciclo respiratório e a pausa inspiratória foi ajustada em $10 \%$ do tempo total. $\mathrm{AFiO}_{2}$ inicial foi ajustada em 0,6 e alterada de maneira a manter uma saturação de oxigênio à oximetria de pulso maior que $93 \%$. Em cada paciente incluído, uma segunda gasometria foi colhida ao final do tempo de estudo (1 ou 2 horas) e a relação $\mathrm{PaO}_{2} / \mathrm{FiO}_{2}$ e shunt pulmonar foram avaliados. As gasometrias arteriais foram analisadas através de um hemogasímetro.

Inicialmente, o estudo foi planejado para observar o efeito da ventilação com modalidades ventilatórias controladas a pressão e a volume sobre a relação $\mathrm{PaO}_{2} / \mathrm{FiO}_{2}$ e shunt pulmonar após uma hora de ventilação mecânica em pacientes que desenvolviam hipoxemia importante no período pós-operatório imediato. Nessa primeira fase, 32 pacientes foram estudados. Em vista dos resultados observados na análise intermediária, uma segunda parte do estudo foi realizada em seguida para observar os efeitos das modalidades ventilatórias sobre a relação $\mathrm{PaO}_{2} / \mathrm{FiO}_{2}$ e shunt pulmonar após um período de duas horas.

Os resultados do dados demográficos e parâmetros ventilatórios foram comparados entre os grupos ventilados à pressão controlada e volume controlado através de teste T não pareado. Análise de variância de duas vias para medidas repetidas foi utilizada para comparar os valores da relação $\mathrm{PaO}_{2} / \mathrm{FiO}_{2}$ e shunt pulmonar entre os dois grupos. A modalidade ventilatória foi o fator intergrupo e o tempo de ventilação foi fator intragrupo. Foi considerado estatisticamente significante $p<0,05$. Todas as análises estatísticas foram realizadas utilizando-se o programa estatístico Statview 5.0. Os resultados são apresentados com média \pm desvio padrão a menos que esteja especificado.

\section{RESULTADOS}

Sessenta e um pacientes que evoluíram com relação $\mathrm{PaO}_{2} / \mathrm{FiO}_{2}$ menor que 200 no período pós-operatório imediato de cirurgia de revascularização do miocárdio foram con- 
secutivamente estudados. Inicialmente 32 pacientes foram estudados. 16 pacientes foram alocados no grupo VCP e 16 no grupo VCV e comparados após um período de ventilação mecânica de uma hora. Contudo, devido aos resultados iniciais pouco significativos, o estudo foi acrescido de uma segunda parte, na qual outros 16 pacientes foram alocados no grupo VCP e 13 no grupo VCV e os efeitos das modalidades ventilatórias sobre a oxigenação foram comparados após um período de 2 horas de ventilação mecânica. Com exceção da idade dos pacientes incluídos na primeira parte do estudo, as características antropométricas e as variáveis ventilatórias, o shunt pulmonar e a relação $\mathrm{PaO}_{2} / \mathrm{FiO}_{2}$ à admissão não foram estatisticamente diferentes entre os grupos ventilados com VCP e VCV nas duas partes do estudo (Tabelas I e II).

Tabela I - Características Antropométricas, Ventilatórias, Shunt Pulmonar e Relação $\mathrm{PaO}_{2} / \mathrm{FiO}_{2}$ dos Pacientes na Admissão à UTI na Primeira Parte do Estudo (Duração de ventilação mecânica de 1 hora) (Média \pm $\mathrm{DP})$

\begin{tabular}{lcc}
\hline & VCV & VCP \\
\hline Idade (anos) & $66 \pm 9$ & $57 \pm 10$ * \\
Peso (kg) & $70 \pm 15$ & $80 \pm 16$ \\
Shunt pulmonar (\%) & $28 \pm 7$ & $32 \pm 13$ \\
Relação $\mathrm{PaO}_{2} / \mathrm{FiO}_{2}$ & $162 \pm 29$ & $144 \pm 31$ \\
$\mathrm{PaO}_{2}(\mathrm{mmHg})$ & $97 \pm 17$ & $94 \pm 33$ \\
$\mathrm{PaCO}_{2}(\mathrm{mmHg})$ & $38 \pm 5$ & $35 \pm 6$ \\
$\mathrm{SaO}_{2}(\%)$ & $96 \pm 2$ & $94 \pm 4$ \\
$\mathrm{PvO}_{2}(\mathrm{mmHg})$ & $42 \pm 6$ & $39 \pm 10$ \\
$\mathrm{SvO}_{2}(\%)$ & $71 \pm 7$ & $64 \pm 14$ \\
$\mathrm{Hb}^{(\mathrm{g} / \mathrm{dl})}$ & $11 \pm 1$ & $11 \pm 1$ \\
\hline
\end{tabular}

* valor de $p<0,05$
Tabela II - Características Antropométricas, Ventilatórias, Shunt Pulmonar e Relação $\mathrm{PaO}_{2} / \mathrm{FiO}_{2}$ dos Pacientes na Admissão à UTI na Segunda Parte do Estudo (Duração de ventilação mecânica de 2 horas) (Média \pm DP)

\begin{tabular}{lcc}
\hline & VCV & VCP \\
\hline Idade (anos) & $65 \pm 7$ & $64 \pm 11$ \\
Peso (kg) & $71 \pm 20$ & $74 \pm 14$ \\
Shunt pulmonar (\%) & $28 \pm 9$ & $32 \pm 15$ \\
Relação $\mathrm{PaO}_{2} / \mathrm{FiO}_{2}$ & $168 \pm 22$ & $159 \pm 21$ \\
$\mathrm{PaO}_{2}(\mathrm{mmHg})$ & $101 \pm 13$ & $95 \pm 12$ \\
$\mathrm{PaCO}_{2}(\mathrm{mmHg})$ & $37 \pm 6$ & $40 \pm 7$ \\
$\mathrm{SaO}_{2}(\%)$ & $96 \pm 1$ & $95 \pm 1$ \\
$\mathrm{PvO}_{2}(\mathrm{mmHg})$ & $40 \pm 7$ & $44 \pm 11$ \\
$\mathrm{SvO}_{2}(\%)$ & $68 \pm 1$ & $69 \pm 1$ \\
$\left.\mathrm{Hb}^{\mathrm{g}} / \mathrm{dl}\right)$ & $11 \pm 2$ & $10 \pm 2$ \\
\hline
\end{tabular}

Como mostram as figuras $1 \mathrm{a}, 1 \mathrm{~b}, 2 \mathrm{a}$ e $2 \mathrm{~b}$, houve aumento na relação $\mathrm{PaO}_{2} / \mathrm{FiO}_{2}$ e uma diminuição no shunt pulmonar que foram estatisticamente significativas, após períodos de uma e duas horas de ventilação mecânica utilizando VCV e VCP. Contudo, estas alterações da relação $\mathrm{PaO}_{2} / \mathrm{FiO}_{2}$ e do shunt pulmonar comportaram-se da mesma maneira em ambos os grupos.

\section{DISCUSSÃO}

Neste estudo, foi observada uma melhora da oxigenação expressa pelo aumento da relação $\mathrm{PaO}_{2} / \mathrm{FiO}_{2}$ e diminuição do shunt pulmonar após períodos de uma e duas horas em ambas as modalidades ventilatórias utilizadas. Contudo, não foram observadas diferenças significativas na oxigenação entre os grupos ventilados em modalidade controlada a volume e a pressão, quando foram utilizados volume corrente, re-

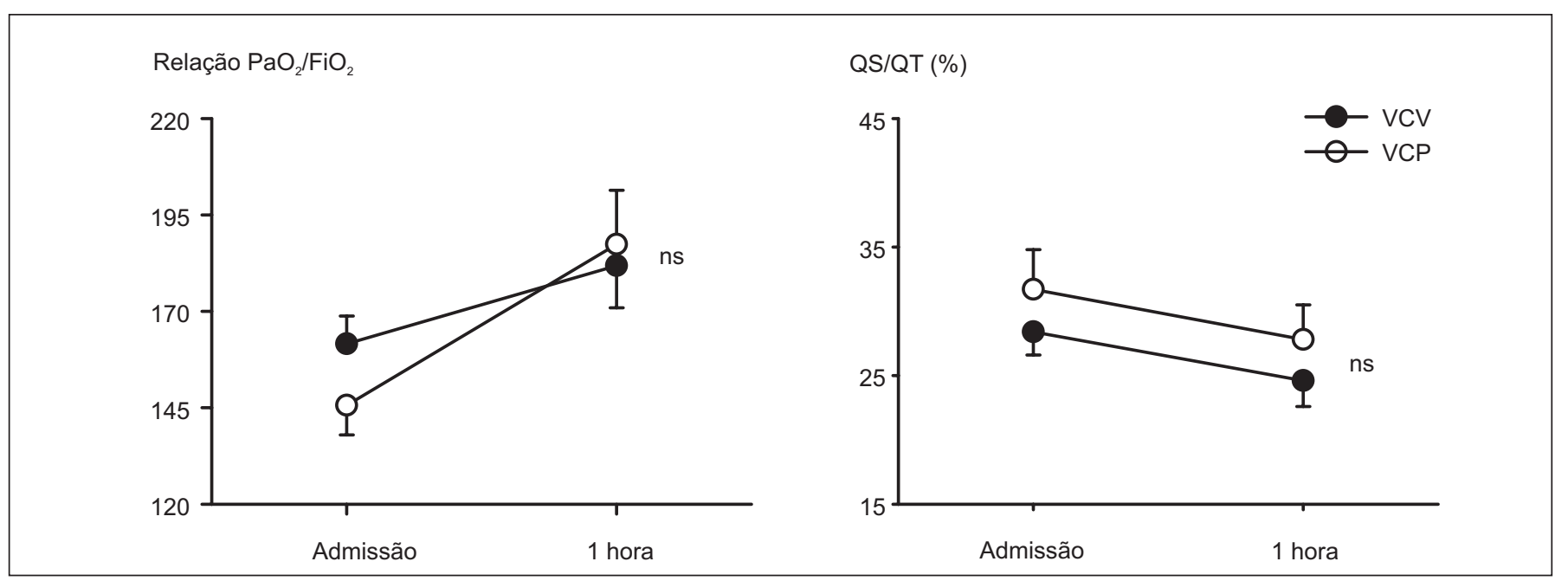

Figuras 1a e 1b - O painel da esquerda (Figura 1a) mostra a relação $\mathrm{PaO}_{2} / \mathrm{FiO}_{2}$ no grupo ventilação controlada a volume (círculos fechados) e no grupo ventilação controlada à pressão (círculos abertos) imediatamente e uma hora após a admissão à unidade de terapia intensiva. O painel da direita (Figura 1b) mostra o shunt pulmonar nos dois grupos. Não foram observadas diferenças entre a relação $\mathrm{PaO}_{2} / \mathrm{FiO}_{2} \mathrm{e} o$ shunt pulmonar entre os grupos nos momentos estudados. Os resultados são expressos como média \pm erro padrão. ns - diferença não significativa. 


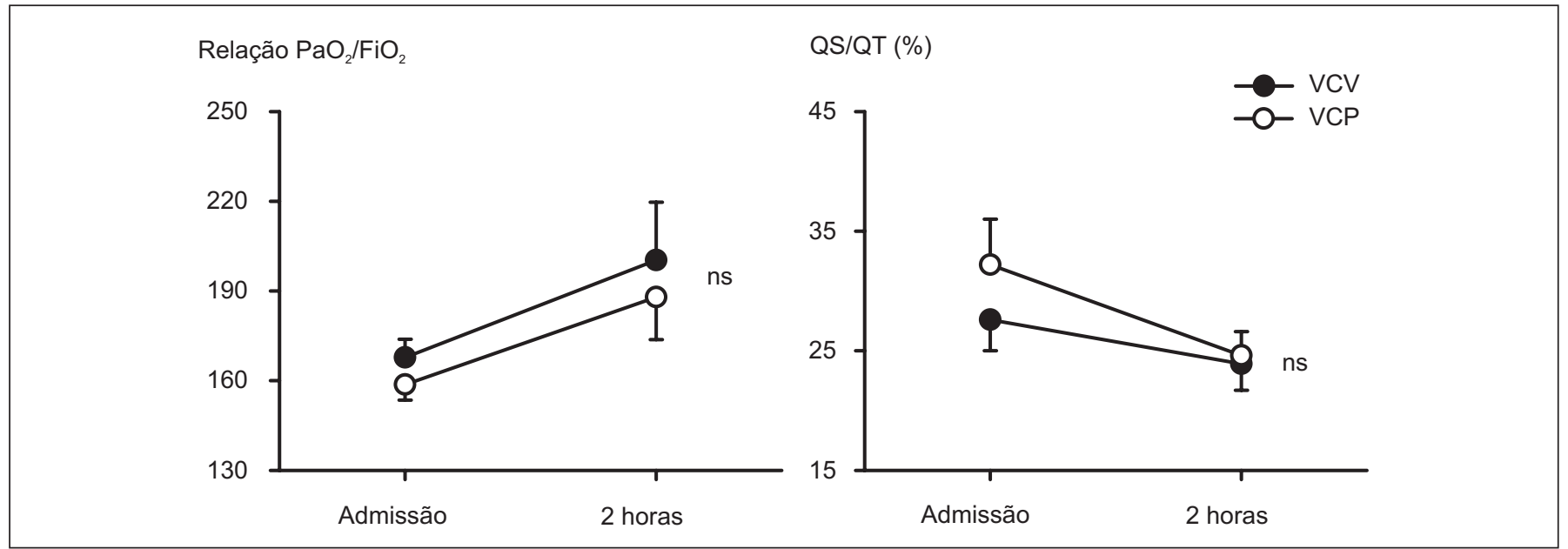

Figuras $2 a$ e $2 b$ - $O$ painel da esquerda (Figura 2a) mostra a relação $\mathrm{PaO}_{2} / \mathrm{FiO}_{2}$ no grupo ventilação controlada a volume (círculos fechados) e no grupo ventilação controlada à pressão (círculos abertos) imediatamente e duas horas após a admissão à unidade de terapia intensiva. O painel da direita (Figura $2 \mathrm{~b}$ ) mostra o shunt pulmonar nos dois grupos. Não foram observadas diferenças entre $\mathrm{F}_{\text {relação }} \mathrm{PaO}_{2} / \mathrm{FiO}_{2}$ e o shunt pulmonar entre os grupos nos momentos estudados. Os resultados são expressos como média \pm erro padrão. ns - diferença não significativa.

lação inspiração/expiração, freqüência respiratória e pressão positiva ao final da expiração iguais nos dois grupos. Hipoxemia é uma complicação freqüentemente observada no período pós-operatório imediato em pacientes submetidos à cirurgia cardíaca com circulação extracorpórea. Num estudo recente realizado em nossa UTI, foi observado que numa série de 61 pacientes consecutivos admitidos à UTI cirúrgica no período pós-operatório imediato de cirurgia cardíaca com circulação extracorpórea, $81 \%$ deles apresentavam uma relação $\mathrm{PaO}_{2} / \mathrm{FiO}_{2}$ menor que 300 à primeira gasometria arterial e $35 \%$ apresentaram uma relação $\mathrm{PaO}_{2} / \mathrm{FiO}_{2}$ menor que $200{ }^{12}$. Foi observada ainda neste estudo uma correlação significativa entre o grau de hipoxemia e a duração da ventilação mecânica no período pós-operatório imediato. Como conseqüência, pode-se esperar aumento da morbidade associada à ventilação mecânica. De acordo com esta hipótese, alguns estudos mostram que o aumento do tempo de duração de ventilação mecânica está correlacionado a um aumento da incidência de lesão pulmonar e infecções respiratórias associadas à ventilação mecânica ${ }^{2,3}$, do tempo de permanência do paciente na unidade de terapia intensiva e dos custos hospitalares, conseqüentemente.

A hipoxemia observada após cirurgia cardíaca com circulação extracorpórea é secundária a fatores mecânicos (atelectasia) e inflamatórios (edema pulmonar de alta permeabilidade). A pressão exercida pelas vísceras abdominais sobre o diafragma relaxado na posição supina ${ }^{14}$, a pressão induzida pelo coração sobre o pulmão ${ }^{15}$ e a manipulação cirúrgica contribuem para formação mecânica de atelectasias principalmente nas regiões pulmonares dependentes e caudais ${ }^{16}$. Associam-se aos fatores mecânicos, a alteração do sistema surfactante após a circulação extracorpórea ${ }^{17}$, aumento da permeabilidade vascular secundária à resposta inflamatória sistêmica induzida pela circulação extracorpórea ${ }^{18}$ e lesão de isquemia e reperfusão que contribuem para formação de edema pulmonar. O edema pulmonar irá determinar um aumento da pressão hidrostática exercida pelo próprio parênquima pulmonar sobre as regiões pulmonares dependentes ${ }^{19}$ e diminuição da difusão do oxigênio por edema alveolar. Em última instância, estas regiões de atelectasia e edema pulmonar irão promover a formação de zonas de shunt intrapulmonar levando à hipoxemia ${ }^{20}$. Do ponto de vista mecânico do sistema respiratório, observa-se diminuição da complacência estática do sistema respiratório e pulmão e presença de ponto de inflexão inferior em curvas pressão volume do sistema respiratório após circulação extracorpórea 21,22

A perda de aeração e o edema se distribuem de maneira heterogênea no parênquima pulmonar, sendo mais importante nas regiões dependentes e próximas do diafragma ${ }^{23,24}$. Isto determina presença de complacências regionais e constantes de tempo diferentes nas diversas regiões pulmonares, com tempo para insuflação alveolar diferentes. Partindo deste pressuposto, a ventilação com pressão controlada, devido ao seu padrão de fluxo característico, seria teoricamente favorável a esta situação, o que permitiria uma insuflação pulmonar mais homogênea e com pressões alveolares mais baixas. Outra vantagem teórica seria a limitação das pressões de vias aéreas ${ }^{25}$, diminuindo as lesões pulmonares induzidas pela ventilação mecânica. Devido a isto, a ventilação com pressão controlada tem sido utilizada em nossa UTI como modalidade ventilatória de escolha na presença de importante hipoxemia, seguindo-se à circulação extracorpórea.

Contudo, nenhum estudo foi capaz de demonstrar vantagens claras da VCP em relação à ventilação controlada a volume no contexto de insuficiência respiratória aguda de causas diversas. Este estudo foi planejado para testar o efeito das modalidades de administração do fluxo inspiratório na VCP e VCV sobre as variáveis de oxigenação, sem que hou- 
vesse interferências de outras técnicas adjuntas da ventilação mecânica, como pressão positiva ao final da inspiração ou manobras de recrutamento alveolar, quando o volume corrente, relação inspiração/expiração e freqüência respiratória foram fixados em ambos os grupos.

Os resultados do presente estudo mostraram que não existem diferenças no padrão de melhora da oxigenação, quando diferentes modalidades de fluxo inspiratório são aplicadas. Contudo, devido à curta duração da hipoxemia observada no período pós-operatório, não nos foi possível avaliar se existem diferenças entre as modalidades ventilatórias e se são efetivas em recrutar áreas totalmente ou parcialmente colapsadas do pulmão. No entanto, os resultados deste estudo estão de acordo com os resultados observados em outros estudos, comparando VCP e VCV no tratamento de síndrome do desconforto respiratório agudo de diversas etiologias ${ }^{5}$.

Esta investigação foi inicialmente proposta para verificar os efeitos de duas modalidades ventilatórias sobre a oxigenação durante uma hora no período pós-operatório imediato em pacientes com hipoxemia importante. Como pode ser observado nas figuras $1 \mathrm{a}$ e $1 \mathrm{~b}$, apesar da ausência de diferença estatisticamente significativa, houve uma tendência para aumento mais acentuado da relação $\mathrm{PaO}_{2} / \mathrm{FiO}_{2}$ no grupo ventilado a pressão em relação ao grupo ventilado a volume. Devido a estes resultados iniciais, uma nova série de pacientes consecutivos com hipoxemia ventilados com VCP ou VCV foi observada. Foi decidido por realizar uma nova coleta de dados, observando-se duas horas de ventilação. Este período foi escolhido, pois os pacientes ainda estariam sob assistência ventilatória mecânica e poderiam mostrar curvas de tendência da relação $\mathrm{PaO}_{2} / \mathrm{FiO}_{2}$ e shunt pulmonar em respostas às modalidades ventilatórias estudadas; isto porque a hipoxemia após cirurgia cardíaca é principalmente secundária à atelectasia e apresenta uma reversão rápida quando comparada à diminuição da oxigenação observada em outras formas de insuficiência respiratória. Períodos de ventilação mais longos implicariam a necessidade de sedação adicional, o que poderia retardar a extubação.

Outro aspecto metodológico a ser considerado neste trabaIho é a impossibilidade de realizar estudo encoberto. É impossível prever antes da cirurgia que pacientes irão desenvolver hipoxemia importante no pós-operatório imediato. Isto implica que os pacientes sejam incluídos após a admissão à UTI, e o responsável esteja de acordo com a sua inclusão no estudo.

Em conclusão, este estudo mostrou que as modalidades ventilatórias controladas a volume e pressão são igualmente eficientes no tratamento de hipoxemia observada no período pós-operatório imediato de cirurgia de revascularização do miocárdio, em pacientes sem doenças pulmonares prévias ou complicações hemodinâmicas.

\section{Comparison between Pressure Controlled and Controlled Mandatory Ventilation in the Treatment of Postoperative Hypoxemia after Myocardial Revascularization}

Fábio Bonini Castellana, M.D., Luiz Marcelo Sá Malbouisson, TSA, M.D., Maria José Carvalho Carmona, TSA, M.D., Célia Regina Lopes, M.D., José Otávio Costa Auler Júnior, TSA, M.D.

\section{INTRODUCTION}

Severe hypoxemia characterized by $\mathrm{PaO}_{2} / \mathrm{FiO}_{2}$ ratio lower than 200 in the immediate postoperative period of myocardial revascularization surgery with cardiopulmonary bypass is a highly lethal complication requiring ventilatory support with positive end expiratory pressure (PEEP) in progressive levels ${ }^{1}$ for longer periods. Longer mechanical ventilation time is related to increase in the incidence of mechanical ventilation-related pneumonias ${ }^{2,3}$. Pressure controlled ventilation (PCV) has been used in our Intensive Care Unit (ICU) as the ventilatory mode of choice for severe postoperative hypoxemia. This is because this ventilatory mode is associated to earlier respiratory system mechanical properties recovery as compared to controlled mandatory ventilation (CMV) during acute respiratory discomfort syndrome $(A R D S)^{4}$ with consequent oxygenation improvement. Other studies, however, have not shown oxygenation improvement or true pulmonary shunt decrease with PCV as compared to $\mathrm{CMV}^{5,6}$.

During CMV inspiratory phase, gas flow is free and decreases in a logarhythmic manner when airway pressure reaches a predetermined value during pulmonary inflation. This modality would have theoretical advantages, such as airway plateau pressure mimicking, leading to a lower incidence of barotraumas and, as a consequence, of mechanical ventilation-induced pulmonary injury in pulmonary parenchyma $^{7-11}$, and a more homogeneous tidal volume distribution respecting regional compliances.

This study aimed at comparing oxygenation evolution in the immediate postoperative period of patients submitted to myocardial revascularization and ventilated under PCV or CMV with slow flow.

\section{METHODS}

After Hospital das Clínicas, Universidade de São Paulo Ethics Committee approval, participated in this study 61 patients submitted to myocardial revascularization with cardiopulmonary bypass. Inclusion criteria were: 1) $\mathrm{PaO}_{2}$ $/ \mathrm{FiO}_{2}$ ratio lower than 200 observed at first blood gases analysis at patient's admission to ICU; 2) lack of previous pulmonary disease; 3 ) lack of major myocardial dysfunction; and 4) lack of bronchopleural fistula. Exclusion criteria were: 1) postoperative circulatory shock needing norepinephrine or dobutamine in doses higher than $10 \mu \mathrm{g} \cdot \mathrm{kg}^{-1} \cdot \mathrm{min}^{-1} ; 2$ ) need for 
mechanical circulatory support; and 3) major postoperative bleeding. Patients' consent to participate in the study was given at ICU admission by their tutors.

Monitoring consisted of electrocardioscopy, radial and pulmonary artery catheter, pulse oximetry, esophageal temperature and capnography. Anesthesia was induced and maintained with adequate doses of midazolam, fentanyl, nondepolarizing neuromuscular blocker and isoflurane. Cardiopulmonary bypass was achieved in normothermia and with membrane oxygenator. Propofol infusion was used during cardiopulmonary bypass to maintain hypnosis and amnesia. Dobutamine, dopamine, nitroglycerin or sodium nitroprusside were used for cardiopulmonary bypass weaning, as needed.

After ICU admission, arterial blood gases were analyzed for each patient. Those with $\mathrm{PaO}_{2} / \mathrm{FiO}_{2}$ ratio lower than 200 were randomly allocated in two groups. One group was ventilated with controlled tidal volume (CTV) and constant inspiratory flow, and the other group was pressure controlled ventilated (PCV) using mechanical ventilators. For both groups, tidal volume was adjusted to $7 \mathrm{ml}^{\mathrm{kg}}{ }^{-1}$, respiratory rate was maintained in $12 \mathrm{ipm}$ and positive end expiratory pressure was maintained in $5 \mathrm{cmH}_{2} \mathrm{O}$. Inspiratory time represented $33 \%$ of total respiratory cycle time and inspiratory pause was adjusted to $10 \%$ of total time. Initial $\mathrm{FiO}_{2}$ was set in 0.6 and changed to maintain oxygen saturation above $93 \%$. Asecond blood gases analysis was performed for each selected patient at the end of the study ( 1 or 2 hours) and $\mathrm{PaO}_{2} / \mathrm{FiO}_{2}$ ratio and pulmonary shunt were evaluated. Arterial blood gases were analyzed through a hemogasometer.

The study was initially designed to observe the effects of pressure or mandatory controlled ventilation on $\mathrm{PaO}_{2} / \mathrm{FiO}_{2}$ ratio and pulmonary shunt after one hour of mechanical ventilation in patients developing severe immediate postoperative period hypoxemia. In this first stage, 32 patients were evaluated. Faced to intermediate analysis results, a second stage of the study was then performed to observe the effects of ventilatory modes on $\mathrm{PaO}_{2} / \mathrm{FiO}_{2}$ ratio and pulmonary shunt after a 2-hour period.

Demographic and ventilatory parameters results were compared between pressure and mandatory controlled ventilation groups through the unpaired Student's $t$ test. Two-way analysis of variance for repeated measures was used to compare $\mathrm{PaO}_{2} / \mathrm{FiO}_{2}$ ratio and pulmonary shunt between groups. Ventilatory mode was the intergroup factor and ventilation time was the intragroup factor. Significance level was $p<$ 0.05 . All statistical analysis were performed using the statistical program Statview 5.0. Results are presented as mean \pm standard deviation unless otherwise specified.

\section{RESULTS}

Sixty-one patients with $\mathrm{PaO}_{2} / \mathrm{FiO}_{2}$ ratio lower than 200 in the immediate postoperative period were consecutively studied. Initially, 32 patients were evaluated; 16 patients were allocated to the PCV group and 16 to the MCV group and were compared after 1 hour of mechanical ventilation. However, due to insignificant initial results, a second stage was added to the study where another 16 patients were allocated to the PCV group and 13 patients to the MCV group. The effects of ventilatory modes on oxygenation were compared after a 2-hour period of mechanical ventilation. Except for age of patients included in the first stage, demographic data and ventilatory variables, pulmonary shunt and $\mathrm{PaO}_{2} / \mathrm{FiO}_{2}$ ratio at admission were not statistically different between groups ventilated with PCV and MCV in both parts of the study (Tables I and II).

Table I - Demographics, Ventilatory Characteristics, Pulmonary Shunt and $\mathrm{PaO}_{2} / \mathrm{FiO}_{2}$ Ratio of Patients Admitted to the ICU in the First Stage of the Study (Mechanical Ventilation Duration $=1$ hour). (Mean \pm SD)

\begin{tabular}{lcc}
\hline & $\mathrm{MCV}$ & $\mathrm{PCV}$ \\
\hline Age (years) & $66 \pm 9$ & $57 \pm 10^{*}$ \\
Weight (kg) & $70 \pm 15$ & $80 \pm 16$ \\
Pulmonary shunt (\%) & $28 \pm 7$ & $32 \pm 13$ \\
$\mathrm{PaO}_{2} / \mathrm{FiO}_{2}$ ratio & $162 \pm 29$ & $144 \pm 31$ \\
$\mathrm{PaO}_{2}(\mathrm{mmHg})$ & $97 \pm 17$ & $94 \pm 33$ \\
$\mathrm{PaCO}_{2}(\mathrm{mmHg})$ & $38 \pm 5$ & $35 \pm 6$ \\
$\mathrm{SaO}_{2}(\%)$ & $96 \pm 2$ & $94 \pm 4$ \\
$\mathrm{PvO}_{2}(\mathrm{mmHg})$ & $42 \pm 6$ & $39 \pm 10$ \\
$\mathrm{SvO}_{2}(\%)$ & $71 \pm 7$ & $64 \pm 14$ \\
$\mathrm{Hb}^{(\mathrm{g} / \mathrm{dl})}$ & $11 \pm 1$ & $11 \pm 1$ \\
\hline
\end{tabular}

${ }^{*} p<0.05$

Table II - Demographics, Ventilatory Characteristics, Pulmonary Shunt and $\mathrm{PaO}_{2} / \mathrm{FiO}_{2}$ Ratio of Patients Admitted to the ICU in the Second Stage of the Study (Mechanical Ventilation Duration $=2$ hours). (Mean \pm SD)

\begin{tabular}{lcc}
\hline & $\mathrm{MCV}$ & $\mathrm{PCV}$ \\
\hline Age (years) & $65 \pm 7$ & $64 \pm 11$ \\
Weight (kg) & $71 \pm 20$ & $74 \pm 14$ \\
Pulmonary shunt (\%) & $28 \pm 9$ & $32 \pm 15$ \\
$\mathrm{PaO}_{2,} \mathrm{FiO}_{2}$ ratio & $168 \pm 22$ & $159 \pm 21$ \\
$\mathrm{PaO}_{2}(\mathrm{mmHg})$ & $101 \pm 13$ & $95 \pm 12$ \\
$\mathrm{PaCO}_{2}(\mathrm{mmHg})$ & $37 \pm 6$ & $40 \pm 7$ \\
$\mathrm{SaO}_{2}(\%)$ & $96 \pm 1$ & $95 \pm 1$ \\
$\mathrm{PvO}_{2}(\mathrm{mmHg})$ & $40 \pm 7$ & $44 \pm 11$ \\
$\mathrm{SvO}_{2}(\%)$ & $68 \pm 1$ & $69 \pm 1$ \\
$\mathrm{Hb}_{(\mathrm{g} / \mathrm{dl})}$ & $11 \pm 2$ & $10 \pm 2$ \\
\hline
\end{tabular}

As shown in figures $1 \mathrm{a}, 1 \mathrm{~b}, 2 \mathrm{a}$ and $2 \mathrm{~b}$, there has been an increase in $\mathrm{PaO}_{2} / \mathrm{FiO}_{2}$ ratio and a decrease in pulmonary shunt which were statistically significant, after 1 and 2-hour periods of PCV and MCV mechanical ventilation. However, these changes in $\mathrm{PaO}_{2} / \mathrm{FiO}_{2}$ ratio and pulmonary shunt were similar for both groups. 


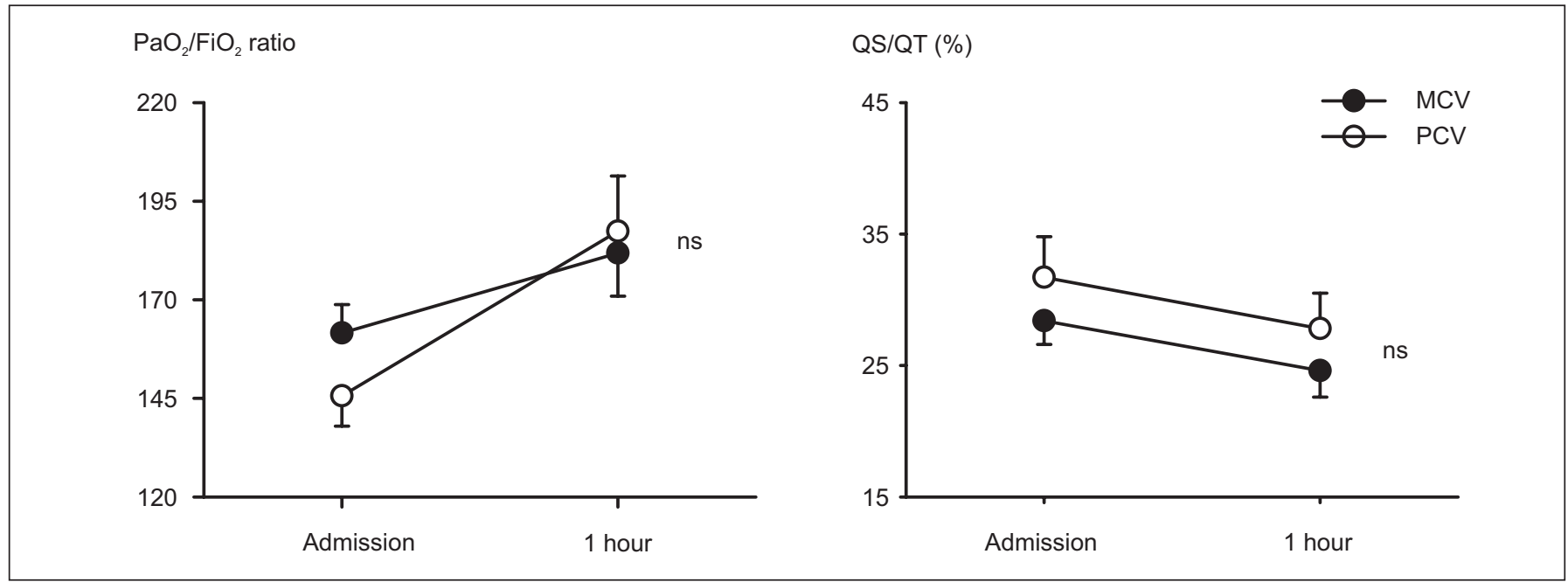

Figures $1 \mathrm{a}$ and $1 \mathrm{~b}$ : The left panel (figure 1a) shows $\mathrm{PaO}_{2} / \mathrm{FiO}_{2}$ ratio in the mandatory controlled ventilation group (full circles) and in the pressure controlled ventilation group (open circles) immediately and one hour after intensive care unit admission. The right panel (figure $1 \mathrm{~b}$ ) shows pulmonary shunt for both groups. There were no differences in $\mathrm{PaO}_{2} / \mathrm{FiO}_{2}$ ratio and pulmonary shunt between groups in the studied moments. Results are in mean \pm SD. ns - no significant difference.

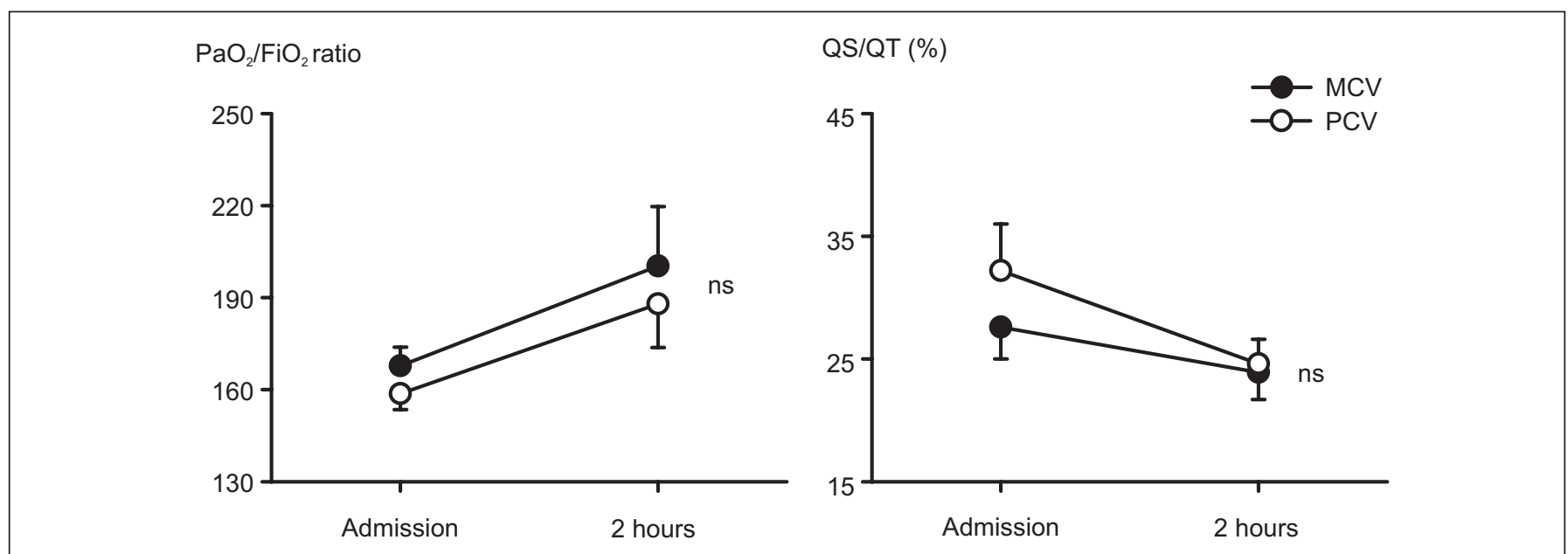

Figures $2 \mathrm{a}$ and $2 \mathrm{~b}$ : The left panel (figure $2 \mathrm{a}$ ) shows $\mathrm{PaO}_{2} / \mathrm{FiO}_{2}$ ratio in the mandatory controlled ventilation group (full circles) and in the pressure controlled ventilation group (open circles) immediately and two hours after intensive care unit admission. The right panel (figure $2 \mathrm{~b}$ ) shows pulmonary shunt for both groups. There were no differences in $\mathrm{PaO}_{2} / \mathrm{FiO}_{2}$ ratio and pulmonary shunt between groups in the studied moments. Results are in mean \pm SD. ns - no significant difference.

\section{DISCUSSION}

Our study has observed an improvement in oxygenation expressed by $\mathrm{PaO}_{2} / \mathrm{FiO}_{2}$ ratio increase and pulmonary shunt decrease after 1 and 2-hour periods in both ventilatory modes. However, there were no significant differences in oxygenation between groups ventilated with PCV and MCV when equal tidal volume, inspiration/expiration ratio, respiratory rate and positive end expiratory pressure were used for both groups.

Hypoxemia is a frequent complication in the immediate postoperative period of patients submitted to cardiac surgery with cardiopulmonary bypass. In a recent study performed in our $I C U$, it was observed that in a series of 61 consecutive patients admitted to ICU in the immediate postoperative period of cardiac surgery with cardiopulmonary bypass, $81 \%$ had $\mathrm{PaO}_{2} / \mathrm{FiO}_{2}$ ratio lower than 300 at first arterial blood gases analysis and $35 \%$ presented $\mathrm{PaO}_{2} / \mathrm{FiO}_{2}$ ratio lower than 200 12. It was also observed a significant correlation between the level of hypoxemia and mechanical ventilation duration in the immediate postoperative period. As a consequence it could be expected an increase in mechanical ventilation-associated morbidity. According to this hypothesis, some studies have shown that longer mechanical ventilation is correlated 
to increase in the incidence of mechanical ventilation-related pulmonary injuries and respiratory infections ${ }^{2,3}$, to longer ICU stay ${ }^{13}$ to and higher hospital costs.

Hypoxemia after cardiac surgery with cardiopulmonary bypass is secondary to mechanical (atelectasis) and inflammatory (pulmonary edema and high patency) factors. Pressure exerted by abdominal viscerae on the relaxed diaphragm in the supine position ${ }^{14}$, heart-induced pressure on the lungs ${ }^{15}$ and surgical manipulation contribute to mechanical development of atelectasis, especially in dependent and caudal pulmonary regions ${ }^{16}$. Changes in surfactant system after cardiopulmonary bypass ${ }^{17}$, vascular patency increase secondary to systemic inflammatory response induced by cardiopulmonary bypass ${ }^{18}$ and ischemia and reperfusion inuries contributing to pulmonary edema are associated to mechanical factors. Pulmonary edema will determine an increase in hydrostatic pressure exerted by the pulmonary parenchyma on dependent pulmonary regions ${ }^{19}$ and a decrease in oxygen spread caused by alveolar edema. Ultimately, these regions of atelectasis and pulmonary edema will form intrapulmonary shunt zones leading to hypoxemia 20. From the respiratory system mechanical point of view, there is a decrease in respiratory system and lungs static compliance and the presence of lower inflection point in respiratory system pressure/volume curve after cardiopulmonary bypass 21,22

The loss of aeration and edema are heterogeneously distributed in the pulmonary parenchyma, being more important in dependent regions close to the diaphragm ${ }^{23,24}$. This determines the presence of regional compliances and different time constants in different pulmonary regions, with different times for alveolar inflation. As from this assumption, pressure controlled ventilation, due to its characteristic flow pattern, would be theoretically favorable to this situation, what would allow a more homogeneous pulmonary inflation with lower alveolar pressures. Another theoretical advantage would be the limitation of airway pressures ${ }^{25}$, decreasing mechanical ventilation-induced pulmonary inuries. For those reasons, pressure controlled ventilation has been used in our ICU as the ventilatory mode of choice in the presence of severe hypoxemia following cardiopulmonary bypass.

However, no study was able to show clear PCV advantages over mandatory controlled ventilation in acute respiratory failures of different causes. This study was planned to test the effect of inspiratory flow administration in PCV and MCV on oxygenation variables without the interference of other adjunct mechanical ventilation techniques, such as positive end expiratory pressure our alveolar recruitment maneuvers when tidal volume, inspiration/expiration ratio and respiratory rate were fixed for both groups.

Our study has shown that there are no differences in oxygenation improvement when different inspiratory flow modes are applied. However, due to the short duration of postoperative hypoxemia it was impossible to evaluate whether there are differences between ventilatory modes and if they are effective in totally or partially recruiting collapsed pulmonary areas. However, our results are in line with other studies comparing
$\mathrm{PCV}$ and MCV in the treatment of acute respiratory distress syndrome of different etiologies ${ }^{5}$.

This investigation was initially proposed to evaluate the effects of two ventilatory modes on oxygenation during 1 immediate postoperative hour in patients with severe hypoxemia. As shown in figures $1 \mathrm{a}$ and $1 \mathrm{~b}$, in spite of the lack of statistically significant differences, there has been a trend for higher increase in $\mathrm{PaO}_{2} / \mathrm{FiO}_{2}$ ratio in the pressure controlled ventilation group as compared to the mandatory controlled ventilation group. Due to these preliminary results, a new series of consecutive hypoxemic patients ventilated with PCV and MCV was studied. We decided to collect new data after 2 hours of ventilation. This period was chosen because patients would be still under mechanical ventilation and could show $\mathrm{PaO}_{2} / \mathrm{FiO}_{2}$ ratio and pulmonary shunt trend curves in response to the ventilatory modes studied; this because post cardiac surgery hypoxemia is primarily secondary to atelectasis and presents a fast reversion as compared to oxygenation decrease observed in other types of respiratory failure. Longer ventilation periods would imply the need for additional sedation, which could delay extubation.

Another methodological aspect to be considered in this study is the impossibility of performing a blind study. It is impossible to determine before surgery patients who will develop severe postoperative hypoxemia. This means that patients have to be included after ICU admission and that tutors have to agree with their participation in the study.

In conclusion, this study has shown that pressure and mandatory controlled ventilation modes are equally effective to treat postoperative hypoxemia after myocardial revascularization surgery in patients without previous pulmonary diseases or hemodynamic complications.

\section{REFERÊNCIAS - REFERENCES}

01. Auler Jr JOC, Carmona MJ, Barbas CV et al - The effects of positive end-expiratory pressure on respiratory system mechanics and hemodynamics in postoperative cardiac surgery patients. Br J Med Biol Res, 2000;33:31-42.

02. Rouby JJ - Histology and microbiology of ventilator-associated pneumonia's. Semin Respir Infect, 1996;11:54-61.

03. Artigas AT, Bello Dronda S, Chacon Valles E et al - Risk factors for nosocomial pneumonia in critically ill trauma patients. Crit Care Med, 2001;29:304-309.

04. Rappaport SH, Shpiner R, Yoshihara G et al - Randomized, prospective trial of pressure-limited versus volume-controlled ventilation in severe respiratory failure. Crit Care Med, 1994;22: 22-32.

05. Esteban A, Alia I, Gordo F et al - Prospective randomized trial comparing pressure-controlled ventilation and volume-controlled ventilation in ARDS. For the Spanish Lung Failure Collaborative Group. Chest, 2000;117:1690-1696.

06. Munoz J, Guerrero JE, Escalante JL et al - Pressure-controlled ventilation versus controlled mechanical ventilation with decelerating inspiratory flow. Crit Care Med, 1993;21:1143-1148.

07 . Dreyfuss D, Soler P, Basset G et al - High inflation pressure pulmonary edema. Respective effects of high airway pressure, high tidal volume, and positive end-expiratory pressure. Am Rev Resp Dis, 1988;137:1159-1164. 
08. Dreyfuss D, Saumon G - Overexpansion pulmonary edema. J Appl Physiol, 1991;71:777-778.

09. Dreyfuss D, Saumon G - Synergistic interaction between alveolar flooding and distention during mechanical ventilation. Am J Respir Crit Care Med, 1996;153:(Suppl):A12.

10. Dreyfuss D, Saumon G - Ventilator-induced lung injury: lessons from experimental studies. Am J Respir Crit Care Med, 1998;157:294-323.

11. Amato MB, Barbas CS, Medeiros DM et al - Effect of a protective-ventilation strategy on mortality in the acute respiratory distress syndrome. N Engl J Med, 1998;338:347-354.

12. Beck K, Malbouisson LMS, Hueb AC et al - Tempo para extubação no pós-operatório de cirurgia cardíaca: correlação entre tempo para extubação e duração de circulação extracorpórea, hidratação per-operatória e relação $\mathrm{PaO}_{2} / \mathrm{FiO}_{2}$. Rev Bras Anestesiol, 1998;48:CBA.

13. Markowicz P, Wolff M, Djedaini K et al - Multicenter prospective study of ventilator-associated pneumonia during acute respiratory distress syndrome. Incidence, prognosis, and risk factors. ARDS Study Group. Am J Respir Crit Care Med, 2000;161: 1942-1948.

14. Froese AB, Bryan AC - Effects of anesthesia and paralysis on diaphragmatic mechanics in man. Anesthesiology, 1974;41: 242-254.

15. Malbouisson LM, Busch CJ, Puybasset L et al - Role of the heart in the loss of aeration characterizing lower lobes in acute respiratory distress syndrome. CT Scan ARDS Study Group. Am J Respir Crit Care Med, 2000;161:2005-2012.

16. Tenling $\mathrm{A}$, Hachenberg $\mathrm{T}$, Tyden $\mathrm{H}$ et al - Atelectasis and gas exchange after cardiac surgery. Anesthesiology, 1998;89: 371-378.

17. Guest Jr JI, Sekulic SM, Yeh TJ et al - Role of atelectasis in surfactant abnormalities following extracorporeal circulation. A clinical and experimental study. Circulation, 1966;33:165-170.

18. Pizov R, Weiss YG, Oppenheim-Eden A et al - High oxygen concentration exacerbates cardiopulmonary bypass-induced lung injury. J Cardiothorac Vasc Anesth, 2000;14:519-523.

19. Pelosi P, D'andrea L, Vitale $G$ et al - Vertical gradient of regional lung inflation in adult respiratory distress syndrome. Am J Respir Crit Care Med, 1994;149:8-13.

20. Magnusson L, Zemgulis V, Wicky S et al - Atelectasis is a major cause of hypoxemia and shunt after cardiopulmonary bypass: an experimental study. Anesthesiology 1997;87:1153-1163.

21. Auler Jr JOC, Zin WA, Caldeira MP et al - Pre and postoperative inspiratory mechanics in ischemic and valvular heart disease. Chest, 1987;92:984-990.

22. Ranieri VM, Vitale N, Grasso S et al - Time-course of impairment of respiratory mechanics after cardiac surgery and cardiopulmonary bypass. Crit Care Med, 1999;27:1454-1460.

23. Hachenberg $T$, Lundquist $H$, Tokics $L$ et al - Analysis of lung density by computed tomography before and during general anaesthesia. Acta Anaesthesiol Scand, 1993;37:549-555.
24. Malbouisson LM, Muller JC, Constantin JM et al - Computed tomography assessment of positive end-expiratory pressure-induced alveolar recruitment in patients with acute respiratory distress syndrome. Am J Respir Crit Care Med, 2001;163: 1444-1450.

25. Auler Jr JOC, Carmona MJC, Silva MH et al - Haemodynamic effects of pressure-controlled ventilation versus volume-controlled ventilation in patients submitted to cardiac surgery. Clin Intensive Care, 1995;6:100-106.

\section{RESUMEN}

Castellana FB, Malbouisson LMS, Carmona MJC, Lopes CR, Auler Jr JOC - Comparación entre Ventilación Controlada a Volumen y a Presión en el Tratamiento de la Hipoxemia en el Período Pos-Operatorio de Cirugía de Revascularización del Miocardio

JUSTIFICATIVA Y OBJETIVOS: Ventilación controlada a presión ha sido utilizada como modalidad ventilatoria de elección en pacientes que desenvuelven hipoxemia importante en el período pos-operatorio de cirugía de revascularización del miocardio. No obstante, no existen estudios mostrando que la ventilación controlada a presión es más efectiva en la reversión de la hipoxemia pos-operatoria que ventilación controlada a volumen. El objetivo de este estudio fue comparar los efectos de ventilación controlada a presión y ventilación controlada a volumen sobre la oxigenación sistémica en pacientes que desenvuelven hipoxemia caracterizada por una relación $\mathrm{PaO}_{2} / \mathrm{FiO}_{2}$ menor que 200 en un período pos-operatorio inmediato de cirugía cardíaca.

MÉTODO: Sesenta y un pacientes con relación $\mathrm{PaO}_{2} / \mathrm{FiO}_{2}$ menor que 200 fueron alocados en un grupo sometido a la ventilación controlada a presión y otro a volumen. El volumen corriente, la frecuencia respiratoria, la relación inspiración/expiración y la presión positiva al final de la expiración fueron las mismas en los dos grupos. Después de la admisión en la UTI y después de períodos de 1 ó 2 horas de ventilación mecánica, la relación $\mathrm{PaO}_{2} / \mathrm{FiO}_{2}$ y el shunt pulmonar fueron cuantificados.

RESULTADOS: Hubo un aumento significativo en la relación $\mathrm{PaO}_{2} / \mathrm{FiO}_{2}$ y una diminución significativa en el shunt pulmonar después de 1 ó 2 horas de ventilación mecánica; sin embargo no fueron observadas diferencias entre las modalidades ventilatorias.

CONCLUSIONES: Las modalidades ventilatorias controladas a volumen y presión fueron igualmente eficientes en el tratamiento de la hipoxemia observada en pacientes en el pos-operatorio inmediato de cirugía de revascularización del miocardio, mostrando que la forma de administración del flujo inspiratorio es poco relevante para el tratamiento de la hipoxemia pos-operatoria. 